\title{
OSTEOMYELITIS AND PATHOLOGICAL FRACTURE OF TIBIA SECONDARY TO PEMPHIGUS VULGARIS
}

Shailesh Kumar K. N1

\section{HOW TO CITE THIS ARTICLE:}

Shailesh Kumar K. N. "Osteomyelitis and Pathological Fracture of Tibia Secondary to Pemphigus Vulgaris". Journal of Evolution of Medical and Dental Sciences 2015; Vol. 4, Issue 57, July 16; Page: 10010-10013, DOI: $10.14260 /$ jemds/2015/1447

\begin{abstract}
We would like to present one rare and interesting case of a patient who suffered osteomyelitis and pathological fracture of tibia due to direct spread of infection from a skin lesion of pemphigus vulgaris. Patient had to suffer chronic osteomyelitis and pathological fracture due to delay in diagnosis and management. He had to undergo multiple surgeries before he could be relieved of his symptoms. Time and again it proves that osteomyelitis is always a clinical diagnosis and it is diagnosed by suspicion. By the time osteomyelitis becomes evident by imaging studies it would have caused significant damage to the host bone.
\end{abstract}

KEYWORDS: Osteomyelitis, Pathological fracture, Sequestrum.

INTRODUCTION: Pathological fracture of long bones following endogenous (haematogenous) osteomyelitis is not uncommon in developing and under developed countries. However pathological fracture following suppurative osteomyelitis secondary to generalized skin disease like pemphigus vulgaris is probably unheard off.

CASE HISTORY: 52 year old man presented with multiple discharging sinuses and inability to bear weight on his right leg from six months. Patient was diagnosed with Pemphigus vulgaris one year back. Patient was on oral steroids and immune modulators for 6 months. During this, a huge vesicle resulted in necrosis of skin over anteromedial aspect of right leg measuring about $15 \times 7 \mathrm{~cm}$ for which he underwent free muscle flap surgery. Within one month of the surgery, multiple discharging sinuses appeared on this flap. Patient was treated with antibiotics and regular dressing for this.

Three months later patient was diagnosed with chronic osteomyelitis right Tibia with pathological fracture for pain on his right leg following a trivial fall. This disabled him from weight bearing on right leg.

During this, patient consulted the author. Examination revealed multiple active sinuses over the right leg and abnormal painful movement at the junction of proximal and mid third of tibia. (Fig. 1)

Intraoperatively, infective debris with purulent collection was noted at the fracture site. Infective debris was curetted and Tibia was stabilized with external fixator. Pus culture showed no growth. (Fig. 2)

In three months, X-ray showed callus over posterolateral cortex and a sequestrum in the proximal fragment. Skin condition improved except a sinus over the sequestrum. Hence Ex-fix was removed and Tibia was put on interlocking nail. Sequestrectomy was deferred for three months fearing the instability due to bone defect. (Fig. 3 and 4)

In one year, patient was able to walk unaided, squat and sit cross legged. Knee movements were full and ankle had $10^{\circ}$ restriction. Except sequestrectomy defect, fracture united completely. (Fig. 5 and 6) 
DISCUSSION: In adults, osteomyelitis is rare and secondary to open fractures or following surgical intervention.1,2 Osteomyelitis in long bones remains challenging despite modern medical technology. $1,3,4$ Chronic skin lesions resulting in osteomyelitis and pathological fracture is rare..$^{5,6} \mathrm{In}$ this case, osteomyelitis of Tibia was due to direct spread of infection from an infective focus. ${ }^{7}$ Patient had to suffer pathological fracture because of the delay in diagnosis and management.1,4,6 Tissues cultured did not show any growth questioning the antibiotic to be used. ${ }^{8}$

Conventionally, an interval of three weeks is required between external fixator removal and nailing. In our case, nailing was done immediately due to poor skin condition ${ }^{1}$. Sequestrum removal was delayed as it may result in significant defect.9,10

CONCLUSION: Acute osteomyelitis is often diagnosed based on clinical suspicion. The delay in diagnosis can result in complications. It is essential that any lesion over a subcutaneous bone should be evaluated for osteomyelitis. Moreover, early sequestrectomy might result in significant defect compelling the surgeon for bone transport thereby prolonging the treatment period.

\section{REFERENCES:}

1. Lazzarini Luca, Mader Jon T, Calhoun Jason H. Osteomyelitis in Long Bones. J Bone Joint Surg Am, 2004 Oct; 86(10): 2305-2318.

2. The in R etal. Delay in diagnosis of femoral hematogenous osteomyel it is in adults: an elusive disease with poor outcome. IMAJ Feb 2013; 15 (2): 85-88.

3. Patwardhan Sandeep et al. Reconstruction of Bone Defects after Osteomyelitis with Nonvascularized Fibular Graft: A Retrospective Study in Twenty-six Children. J Bone Joint Surg Am, 2013 May 01; 95(9): e56.

4. Termaat MF et al. The Accuracy of Diagnostic Imaging for the Assessment of Chronic Osteomyelitis: A Systematic Review and Meta-Analysis. J Bone Joint Surg Am, 2005 Nov; 87(11): 2464-2471.

5. Prose NS, Fahrner LJ, Miller CR, Layfield L. Pustularpsorias is with chronic recurrent multifocal osteomyelitis and spontaneous fractures. Journal of American Academy of dermatology. 1994 Aug; 31 (2 Pt 2): 376-379.

6. Capener Norman, Pierce Kenneth C. Pathological Fractures in Osteomyelitis. J Bone Joint Surg Am, 1932 Jul; 14(3): 501-510.

7. Robertson DE. Acute Hematogenous Osteomyelitis. J Bone Joint Surg Am, 1938 Jan; 20(1): 35-47.

8. Perry CR, Pearson RL, Miller GA. Accuracy of cultures of material from swabbing of the superficial aspect of the wound and needle biopsy in the preoperative assessment of osteomyelitis. J Bone Joint Surg Am, 1991 Jun; 73(5): 745-749.

9. Eralp Levent, Kocaoglu Mehmet, Rashid Haroon. Reconstruction of Segmental Bone Defects Due to Chronic Osteomyelitis with Use of an External Fixator and an Intramedullary Nail Surgical Technique. J Bone Joint Surg Am, 2007 Sep; 89(2 suppl 2): 183-195.

10. Kiter E, Akkaya S, O to M, Günal I. Spontaneous regeneration of the large femoral defect in patient with diffuse osteomyelitis after intramedullary nailing. Joint Diseases and Related Surgery 2010: 21(3): 178-181. 


\section{CASE REPORT}

Fig.1: Preoperative MRI picture of right tibia showing intramedullary collection and pathological fracture.

Fig. 2: Immediate post-op X ray showing tibia on external fixator.

Fig. 3: X-ray picture after 3 months showing callus formation.

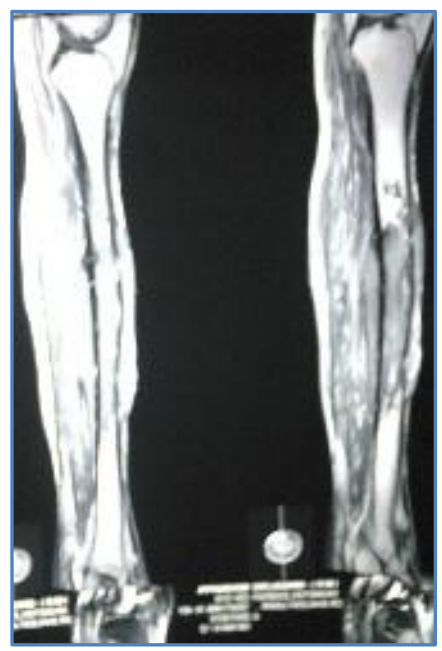

Fig. 1

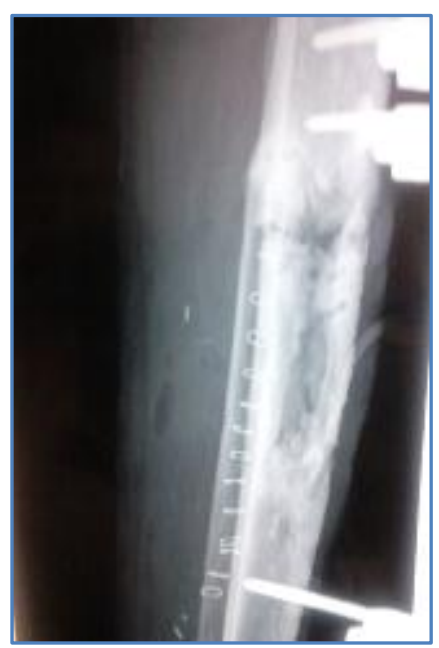

Fig. 2

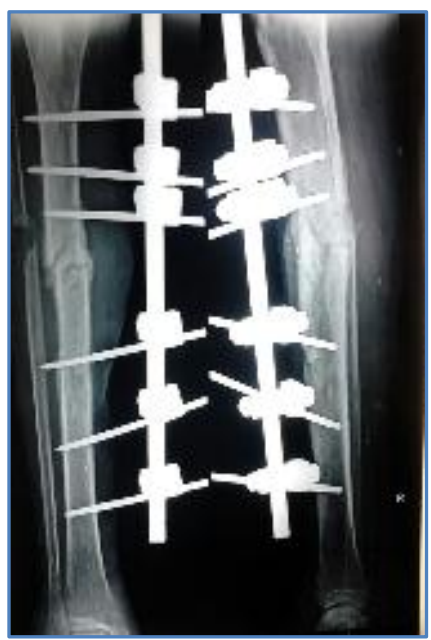

Fig. 3

Fig. 4: X- ray picture after interlocking nailing.

Fig. 5: Six months of nailing showing fracture consolidation and a sequestrum.

Fig. 6: Eight months $x$ ray picture after sequestrectomy.

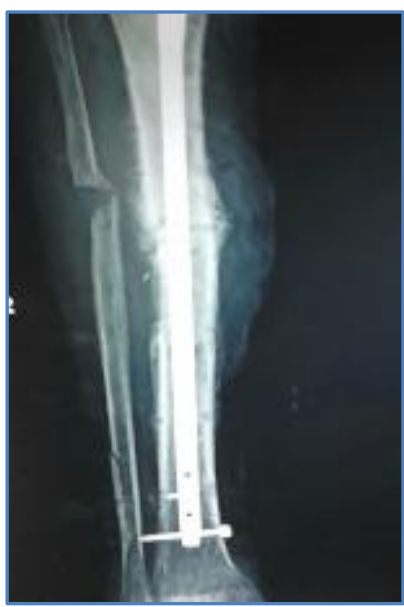

Fig. 4

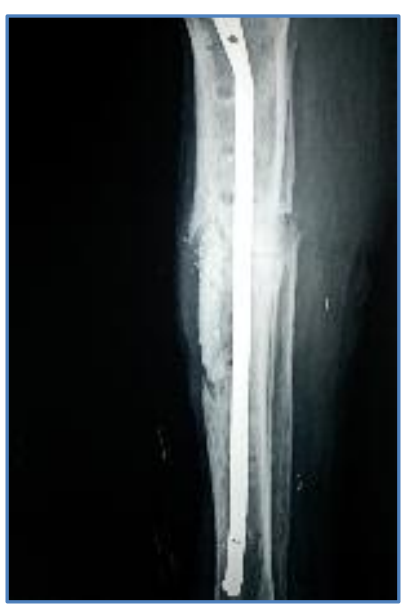

Fig. 5

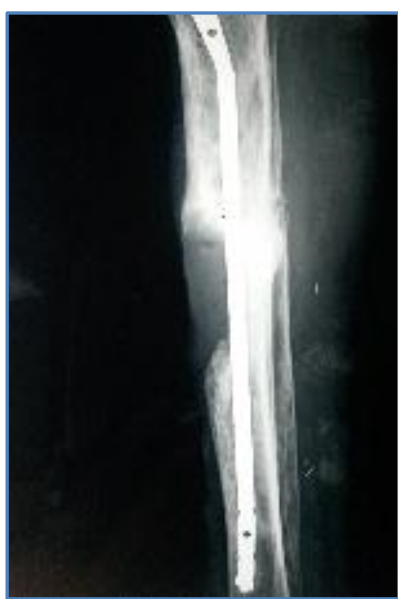

Fig. 6 


\section{CASE REPORT}

\section{AUTHORS:}

1. Shailesh Kumar K. N

\section{PARTICULARS OF CONTRIBUTORS:}

1. Assistant Professor, Department of Orthopaedics, Yenepoya Medical College \& Hospital, Mangalore.

FINANCIAL OR OTHER COMPETING INTERESTS: None

\section{NAME ADDRESS EMAIL ID OF THE} CORRESPONDING AUTHOR:

Dr. Shailesh Kumar K. N, Yashodamma Nilaya, Subhas Nagar, Pandeshwar, Mangalore.

E-mail: shaileshortho2000@yahoo.com

Date of Submission: 21/06/2015. Date of Peer Review: 22/06/2015. Date of Acceptance: 09/07/2015. Date of Publishing: 16/07/2015. 